\title{
Advanced glycation endproducts and their receptor in different body compartments in COPD
}

\author{
Susan J. M. Hoonhorst ${ }^{1,2}$, Adèle T. Lo Tam Loi ${ }^{3}$, Simon D. Pouwels ${ }^{1,2,5}$, Alen Faiz ${ }^{2,5}$, Eef D. Telenga ${ }^{1,2}$, \\ Maarten van den Berge ${ }^{1,2}$, Leo Koenderman ${ }^{3}$, Jan-Willem J. Lammers ${ }^{3}$, H. Marike Boezen ${ }^{2,4}$, \\ Antoon J. M. van Oosterhout ${ }^{2,5}$, Monique E. Lodewijk $k^{2,5}$, Wim Timens ${ }^{2,5}$, Dirkje S. Postma ${ }^{1,2}$ \\ and Nick H. T. ten Hacken ${ }^{1,2^{*}}$
}

\begin{abstract}
Background: Chronic obstructive pulmonary disease (COPD) is a chronic lung disease characterized by chronic airway inflammation and emphysema, and is caused by exposure to noxious particles or gases, e.g. cigarette smoke. Smoking and oxidative stress lead to accelerated formation and accumulation of advanced glycation end products (AGEs), causing local tissue damage either directly or by binding the receptor for AGEs (RAGE). This study assessed the association of AGEs or RAGE in plasma, sputum, bronchial biopsies and skin with COPD and lung function, and their variance between these body compartments.

Methods: Healthy smoking and never-smoking controls $(n=191)$ and COPD patients $(n=97$, GOLD stage I-IV) were included. Autofluorescence (SAF) was measured in the skin, AGEs (pentosidine, CML and CEL) and SRAGE in blood and sputum by ELISA, and in bronchial biopsies by immunohistochemistry. eQTL analysis was performed in bronchial biopsies.

Results: COPD patients showed higher SAF values and lower plasma SRAGE levels compared to controls and these values associated with decreased lung function ( $p<0.001$; adjusting for relevant covariates). Lower plasma sRAGE levels significantly and independently predicted higher SAF values $(p<0.001$ ). One SNP (rs2071278) was identified within a region of $50 \mathrm{kB}$ flanking the AGER gene, which was associated with the gene and protein expression levels of AGER and another SNP (rs2071278) which was associated with the accumulation of AGEs in the skin.

Conclusion: In COPD, AGEs accumulate differentially in body compartments, i.e. they accumulate in the skin, but not in plasma, sputum and bronchial biopsies. The association between lower sRAGE and higher SAF levels supports the hypothesis that the protective mechanism of SRAGE as a decoy-receptor is impaired in COPD.
\end{abstract}

Keywords: COPD, RAGE, Advanged glycation end-products, sRAGE

\section{Background}

Chronic obstructive pulmonary disease (COPD) is characterized by chronic airflow limitation, accompanied by persistent inflammation of the airways, mainly caused by cigarette smoking. Both smoking and inflammation are associated with oxidative stress leading to accelerated

\footnotetext{
* Correspondence: n.h.t.ten.hacken@umcg.nl

'Department of Pulmonary Diseases, University of Groningen, University

Medical Center Groningen, Hanzeplein 1, 9713, GZ, Groningen, The

Netherlands

${ }^{2}$ University of Groningen, University Medical Center Groningen, GRIAC research institute, Groningen, The Netherlands

Full list of author information is available at the end of the article
}

formation and accumulation of advanced glycation end products (AGEs) [1,2].

AGEs are a heterogeneous and complex group of compounds that are irreversibly formed by non-enzymatic glycation and oxidation of proteins and lipids [3]. The formation of AGEs accumulates in tissues with ageing. Furthermore, under oxidative stress and inflammatory conditions their formation and accumulation increase. Therefore, accumulation of AGEs can be used as a readout system for exposure to oxidative stress during life. This is particularly true in tissues with slow turnover, more than in tissues or products from tissues with rapid 
turnover. The best known AGEs are $\mathrm{N}^{\varepsilon}$-(carboxymethyl)lysine (CML), $\mathrm{N}^{\varepsilon}$-(carboxyethyl)lysine (CEL) and pentosidine. AGEs cause local tissue damage by affecting protein structure, by formation of crosslinks between molecules, or by binding the receptor for AGEs (RAGE) $[4,5]$. RAGE is a member of the immunoglobulin superfamily and is a pattern-recognition receptor on cell surfaces. Ligation of RAGE triggers inflammatory responses, induces oxidative stress, and in turn causes RAGE over-expression. This may finally leads to increased tissue remodeling [5]. Interestingly, expression of RAGE in the lung has shown to be relatively high when compared with other tissues [6]. The gene encoding RAGE, AGER, has been shown to be a susceptibility gene for lung function decline and the onset of COPD [7, 8]. Previously, a number of Single Nucleotide Polymorphism (SNP)s within and in close proximity to the AGER gene have been identified to play an important role in its transcriptional and translation regulation (PMID: 23886569). Furthermore, it has been shown that immunostaining of the receptor is increased in bronchial biopsies and lung parenchyma of COPD patients $[9,10]$. Next to AGEs, RAGE can also be activated by endogenous danger signals, or damage associated molecular patterns (DAMPs). Several of these RAGE activating DAMPs, e.g. HMGB1, S100A9 and LL-37 have been shown to be increased in lung fluid or serum from COPD patients compared to smoking and non-smoking controls [11, 12]. Importantly, RAGE also exists as soluble form (sRAGE). It has been postulated that sRAGE can act as a decoy receptor by clearance of circulating AGEs, preventing ligation of membrane bound RAGE. This possible 'protective' mechanism may be reduced in COPD, as levels of sRAGE have found to be lower in COPD patients than in non-COPD controls [13-17].

A few studies have indicated that AGEs are involved in the pathology of COPD. One study showed increased accumulation of AGEs in lung parenchyma and small airways of COPD patients [9]. We and others found increased AGE accumulation in the skin of COPD patients compared to healthy smoking and never-smoking controls $[18,19]$. Furthermore, plasma CML levels in COPD are elevated compared to non-COPD controls [19], suggesting a systemic component that may contribute to AGE accumulation outside the lung, and to extrapulmonary manifestations of COPD.

Taken together, studies so far suggested that the AGERAGE axis is involved in the pathophysiology of COPD. In the current study we evaluated both AGEs and sRAGE levels in plasma, sputum, bronchial biopsies and the skin in the same study subjects. Young (18-40) and old (40-75) smokers and never-smokers, and mild-tovery severe COPD patients were included. We studied whether the expression of AGEs or RAGE in the different tissues was associated with COPD and lung function values, and whether the expression of AGEs was associated with the levels of RAGE in different tissues. Lastly, with a cis-eQTL analysis we investigated the association of SNPs flanking the AGER gene with skin autofluorescence and serum sRAGE levels.

\section{Methods}

Subjects, ethics, consent and permissions

Data were collected from two studies performed in Groningen and Utrecht, the Netherlands (Clinicaltrials.gov: NCT00807469 and NCT00848406 (A multi-center study [20]) and NCT00848406). All participating subjects gave peripheral blood, bronchial brushings and performed an AGE-reader measurement, while a subgroup of subjects underwent sputum induction and bronchoscopy with collection of bronchial biopsies. All measurements were obtained by using standardized protocols. All studies, sample collection and genetic studies were approved by the medical ethics committees of University Medical Centers Groningen (UMCG) and Utrecht (UMCU), the Netherlands. All participants gave their written informed consent.

Mild to very severe COPD patients $(40-75$ years, $>10$ packyears), as classified by the Global Initiative for Chronic Obstructive Lung Disease (GOLD) were recruited from outpatient clinics of UMCG and UMCU. Old (40-75 years) and young (18-40 years) healthy smokers and healthy never-smokers were recruited by advertisements. Old smokers had a smoking history $>10$ packyears and young smokers $>0.5$ packyears. All neversmoking subjects had smoked $<0.5$ packyears. Healthy participants had no history of pulmonary diseases and showed normal spirometry. Exclusion criteria for all groups were alpha-1 antitrypsin deficiency and a doctors' diagnosis of asthma.

\section{Determination of AGEs and RAGE in peripheral blood samples and sputum}

Blood was collected in tubes containing EDTA and was immediately placed on ice. After centrifugation (twice at $2000 \mathrm{rcf}_{\max }, 10 \mathrm{~min}, 4{ }^{\circ} \mathrm{C}$ ) samples were stored at $-80{ }^{\circ} \mathrm{C}$ until analysis. Sputum induction was performed according standard protocols (detailed methods in Additional file 1). Sputum samples were centrifuged $\left(10 \mathrm{~min}, 450 \mathrm{~g}, 4^{\circ} \mathrm{C}\right.$ ) and the supernatant was stored at $-80{ }^{\circ} \mathrm{C}$ until analysis.

In plasma and sputum samples, ELISA was performed to determine levels of total sRAGE (cleaved and secreted forms) (RAGE DuoSet; R\&D Systems, Minneapolis, MN, USA), CEL (Cell Biolabs Inc. San Diego, CA, USA), CML (Cell Biolabs Inc. San Diego, CA, USA) and Pentosidine (Uscn Life Science Inc., Wuhan, China), all according to the manufacturer's instructions. 
Determination of AGEs and RAGE in bronchial biopsies Bronchial biopsies were taken from subsegmental carinae of the right lower lobe. Biopsies were fixed in $4 \%$ neutral buffered formalin, processed and embedded in paraffin and cut in $3 \mu \mathrm{m}$ sections. After antigen retrieval, sections were incubated with the primary monoclonal antibody against AGEs (anti-AGEs (clone 6D12), 1:750, Cosmo Bio Co, Ltd, Tokyo, Japan) or RAGE (anti-RAGE (ab7764), 1:1500, Abcam, Cambridge, UK). Immunohistochemical stainings were performed using the DAKO autostainer (DAKO, Glostrup, Denmark). Quantification of both stainings was performed by calculating the percentage positive and strong positive pixels of the total amount of pixels in whole biopsies, using ImageScope (Aperio Technologies, version 11.2.0.780). Detailed immunohistochemistry and quantification procedures are presented in the Additional file 1.

\section{Measurement of AGEs using Skin autofluorescence in the skin}

Skin autofluorescence (SAF) was assessed non-invasively by the AGE-Reader ${ }^{\text {rux }}$ (DiagnOptics B.V., Groningen, The Netherlands) [21]. Technical details of this device have been extensively described elsewhere and briefly in the Additional file 1 [22]. In short, the volar surface of subject's forearm was positioned on top of the device and three consecutive measurements were performed for each subject. In all analyses, SAF was expressed as the mean of these three measurements in arbitrary units (AU).

\section{Gene expression analysis}

We assessed whether cis-acting SNPs influence the gene and protein expression of AGER and RAGE, respectively. DNA samples were genotyped with Illumina $\mathrm{Hu}-$ man CytoSNP 12 and Illumina OmniExpress Exome. SNPs genotyped in both arrays were select for the eQTL analysis. Gene expression profiles were obtained from bronchial brushing using a Human genome ST v1.0 arrays. Linear regression analysis was used to test for association between the SNPs and 2-log transformed gene expression levels and soluble RAGE measured in sputum and plasma. SNPs were tested in an additive genetic model and the models were adjusted for gender, smoking status and age. A cis-eQTL was defined as a SNP that was significantly associated with gene or protein expression within a $50 \mathrm{kB}$ distance of the AGER gene.

\section{Statistical analysis}

Differences in expression of AGEs and RAGE between groups were analyzed by Kruskal-Wallis test, followed by Mann-Whitney U tests if significant. Associations with COPD were examined by multiple regression analyses with AGEs or RAGE expression as dependent variables, and COPD or lung function values as predictor variables. Associations of AGEs and RAGE between different compartments were additionally analyzed by multiple regression models. All models were adjusted for co-variates that associate with AGE formation, including age, gender, packyears, BMI, LDL cholesterol, and triglycerides. Benjamini Hochberg corrections were applied to correct for multiple testing. Regression models were considered valid if the residuals were normally distributed. Statistical analyses were performed using the statistical program IBM SPSS Statistics version 20.

\section{Results}

\section{Subject characteristics}

In total, 108 young controls (including 36 never-smokers and 72 smokers), 83 old controls (including 28 neversmokers and 55 smokers) and 97 COPD patients (32 GOLD I, 25 GOLD II, 24 GOLD III, 16 GOLD IV) were included. Group characteristics are presented in Table 1.

\section{AGEs}

Expression of AGEs in plasma, sputum, bronchial biopsies and the skin is presented in Fig. 1 and Table 2.

In plasma (Fig. 1a), CEL levels were significantly higher in young healthy subjects compared to old healthy subjects and COPD patients. Furthermore, plasma CML levels were significantly higher in COPD patients compared to young

Table 1 Group characteristics

\begin{tabular}{|c|c|c|c|}
\hline & $\begin{array}{l}\text { Young healthy } \\
n=108\end{array}$ & $\begin{array}{l}\text { Old healthy } \\
n=83\end{array}$ & $\begin{array}{l}\text { COPD } \\
n=97\end{array}$ \\
\hline Age, years & $25(66)$ & $54(8.9)$ & $62(7.6)$ \\
\hline Males, n (\%) & $54(50)$ & $60(72)$ & $68(70)$ \\
\hline Current smokers, n (\%) & $72(67)$ & $54(65)$ & $51(53)$ \\
\hline Cigarettes per day & $9(6.8)$ & $16(7.1)$ & $11(7.9)$ \\
\hline Packyears & $3.1(4.8)$ & $20(18.0)$ & $38(16.8)$ \\
\hline $\mathrm{BMI}, \mathrm{kg} / \mathrm{m}^{2}$ & $23.0(2.8)$ & $25.3(3.6)$ & $25.5(4.7)$ \\
\hline $\mathrm{FEV}_{1}, \%$ pred & $108(9.5)$ & $110(13.5)$ & $62(27)$ \\
\hline $\mathrm{FEV}_{1} / \mathrm{FVC}, \%$ & $85(5.7)$ & $79(5.0)$ & $48(14)$ \\
\hline RV/TLC, \% & $23.3(5.1)$ & $31.1(4.4)$ & $45.8(11.3)$ \\
\hline $\mathrm{FEF}_{25-75}$ \% \%pred & $101(18.7)$ & $100(30.4)$ & $24(17)$ \\
\hline DLCOcNA, \%pred & $97(13.3)$ & 98 (12.6) & $67(24)$ \\
\hline DLCO \%pred & $91.6(12.0)$ & $90.0(12.3)$ & $61.6(23.6)$ \\
\hline LDL cholesterol, mmol/L & $2.6(0.8)$ & $3.6(1.0$ & $3.4(1.0)$ \\
\hline Triglycerides, mmol/L & $1.0(0.8)$ & $1.4(1.0)$ & $1.2(0.7)$ \\
\hline Fasting glucose, $\mathrm{mmol} / \mathrm{L}$ & $5.2(1.3)$ & $5.6(0.7)$ & $5.8(0.7)$ \\
\hline Creatinine, $\mu \mathrm{mol} / \mathrm{L}$ & 73.7 (10.9) & $80.2(14.5)$ & $82.7(14.8)$ \\
\hline
\end{tabular}

Data is presented as mean (sd) unless otherwise stated $n$ number, $B M I$ body mass index, $F E V$, forced expiratory volume in one second, $F V C$ forced expiratory volume, $R V$ residual volume, $T L C$ total lung capacity, FEF forced expiratory flow, $D L C O(C N A)$ diffusion coefficient for carbon monoxide, $L D L$ low density lipoprotein 


\section{a Plasma}
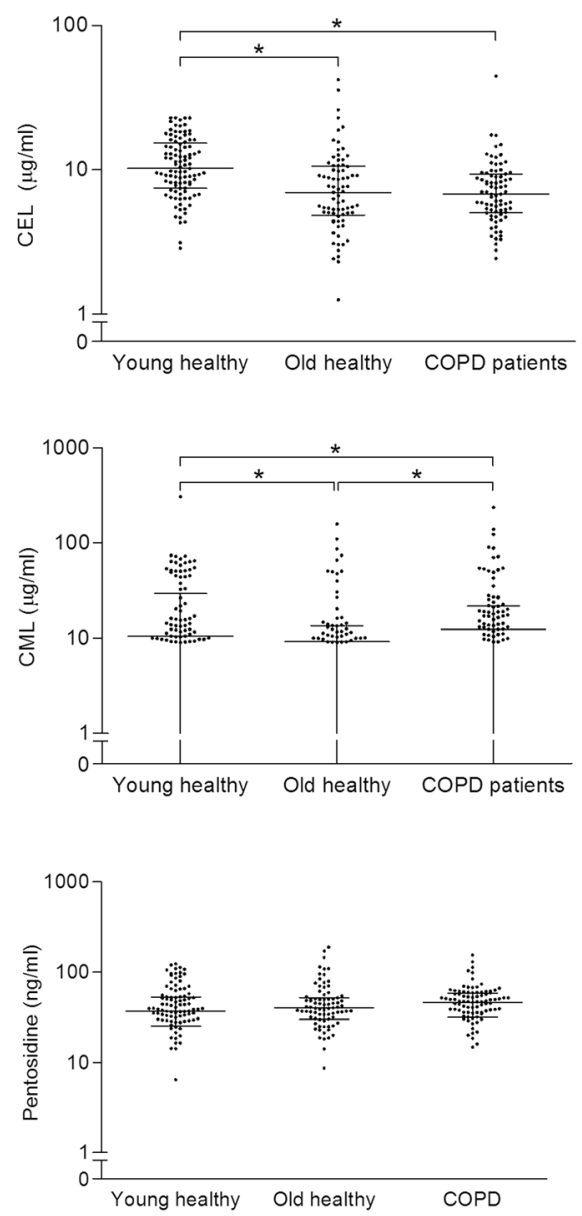

C Bronchial biopsies

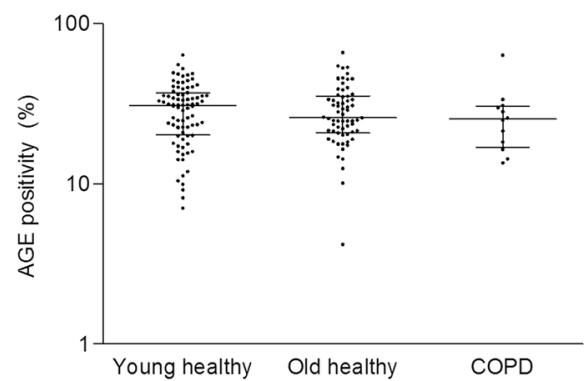

b sputum
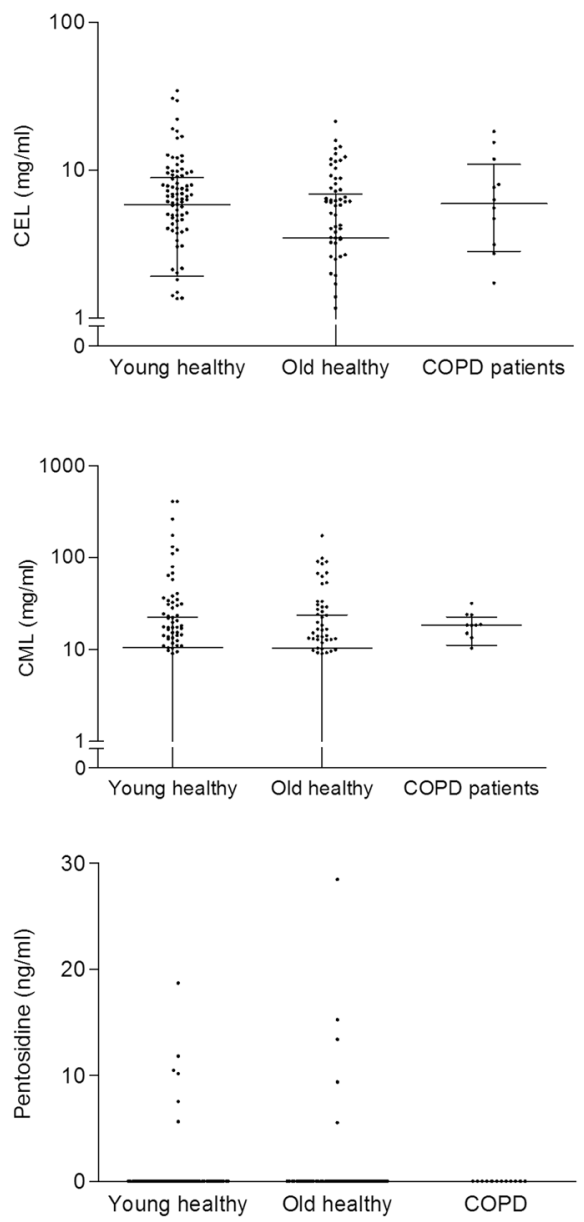

d Skin autofluorescence

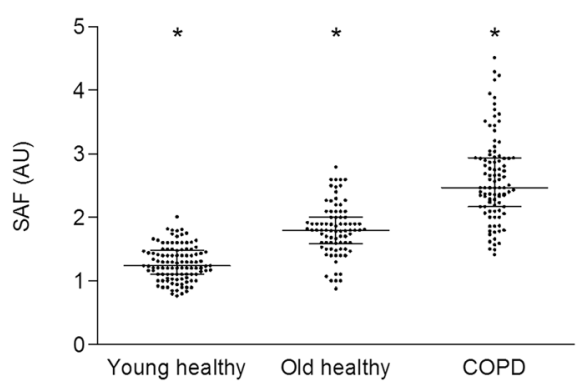

Fig. 1 The expression of AGEs in plasma, sputum, bronchial biopsies and the skin. The levels of AGEs in a plasma, $\mathbf{b}$ sputum, $\mathbf{c}$ bronchial biopsies and $\mathbf{d}$ skin (SAF). Horizontal lines represent median values with interquartile ranges, ${ }^{*} p<0.05$ between groups

and old subjects, and higher in the young group than in the old healthy group. Plasma pentosidine levels did not differ between groups. In sputum (Fig. 1b), CEL and CML levels did not differ between groups, whereas pentosidine levels were too low to be detected; only 11 sputum supernatant samples of the total of 182 samples were above the detection limit of $1.45 \mathrm{ng} / \mathrm{ml}$. AGEs immunopositivity in whole bronchial biopsies was not differently expressed between groups, (Fig. 1c), neither were quantitative analyses in the intact and basal epithelium, smooth muscle and connective tissue (Additional file 1: Figure S1 and S2). However, accumulation of AGEs in the skin was significantly different between all groups, with highest SAF values in COPD patients and lowest values in the young group (Fig. 1d).

In all measurements, the levels of AGEs did not differ between the different stages of COPD (GOLD I-IV, 
Table 2 AGE and RAGE expression in young and old subjects, and COPD patients

\begin{tabular}{|c|c|c|c|c|}
\hline & $\begin{array}{l}\text { Young healthy } \\
<40 \text { years }\end{array}$ & $\begin{array}{l}\text { Old healthy } \\
>40 \text { years }\end{array}$ & $\begin{array}{l}\text { COPD GOLD I-IV } \\
>40 \text { years }\end{array}$ & $\begin{array}{l}\text { Kruskal-Wallis } \\
p \text {-value }\end{array}$ \\
\hline Plasma & $n=105$ & $n=82$ & $n=95$ & \\
\hline $\mathrm{CEL}, \mu \mathrm{g} / \mathrm{ml}$ & $10.2(7.4-15.4)$ & $7.0(4.8-10.6) * *$ & $6.8(5.1-9.4) * *$ & $0.000^{*}$ \\
\hline $\mathrm{CML}, \mu \mathrm{g} / \mathrm{ml}$ & $10.5(0.0-29.5)$ & $9.2(0.0-13.5) * *$ & $12.5(0.0-21.6) * *^{* a}$ & $0.042^{*}$ \\
\hline Pentosidine, ng/ml & $36.8(25.3-53.1)$ & $39.9(30.1-52.0)$ & $46.1(31.8-58.5)$ & 0.181 \\
\hline sRAGE, pg/ml & 795 (614-1089) & 805 (617-1032) & $414(292-592) * * a$ & $0.000^{*}$ \\
\hline Induced sputum & $n=97$ & $n=73$ & $n=12$ & \\
\hline $\mathrm{CEL}, \mu \mathrm{g} / \mathrm{ml}$ & $5.9(1.9-8.9)$ & $3.50(0.0-6.9)$ & $6.0(2.9-11.0)$ & 0.064 \\
\hline $\mathrm{CML}, \mu \mathrm{g} / \mathrm{ml}$ & $10.6(0.0-22.7)$ & $10.4(0.0-23.7)$ & $18.3(11.2-22.5)$ & 0.409 \\
\hline Pentosidine, ng/ml & $0.0(0.0-0.0)$ & $0.0(0.0-0.0)$ & $0.0(0.0-0.0)$ & 0.650 \\
\hline sRAGE, pg/ml & $78.1(0.0-160.2)$ & $114.0(0.0-240.5)$ & 110.9 (49.7-161.8) & 0.117 \\
\hline Bronchial Biopsies & $n=85$ & $n=68$ & $n=12$ & \\
\hline AGEs, positivity (\%) & $31.1(20.3-36.9)$ & $25.9(20.9-35.4)$ & $25.5(16.9-30.7)$ & 0.418 \\
\hline RAGE, positivity (\%) & $9.8(6.5-15.1)$ & $8.2(5.7-11.8)$ & $8.0(4.2-11.4)$ & 0.204 \\
\hline Skin & $n=107$ & $n=83$ & $n=96$ & \\
\hline AGE-reader, SAF & $1.2(1.1-1.5) *$ & $1.8(1.6-2.0) *$ & $2.5(2.2-2.9) *$ & $0.000^{*}$ \\
\hline
\end{tabular}

Data is expressed as median (IQR). ${ }^{*} p<0.05$ between all groups, ${ }^{* *} p<0.05$ compared with the young healthy group, ${ }^{a}$ compared with the old healthy group $C E L \mathrm{~N}^{\varepsilon}$-(carboxyethyl)lysine, $C M L \mathrm{~N}^{\varepsilon}$-(carboxymethyl)lysine, $R A G E$ receptor for advanced glycation endproducts, $A G E s$ advanced glycation endproducts, $S A F$ skin autofluorescence

Additional file 1: Table S1). Additionally, AGE levels are presented separately for healthy never-smokers and smokers (Additional file 1: Table S1).

\section{(s)RAGE}

The levels of RAGE in plasma, sputum and bronchial biopsies are presented in Fig. 2 and Table 2 .

In plasma, sRAGE levels were significantly lower in COPD patients compared to young and old healthy subjects (Fig. 2a). In addition, COPD GOLD stage III patients showed lower sRAGE levels compared to GOLD stage I patients. Furthermore, GOLD stage IV patients showed lower sRAGE levels than GOLD stage I and II patients (Fig. 3a and Additional file 1: Table S1). No differences were found between young and old healthy subjects. RAGE levels in sputum and RAGE immunopositivity in whole sections from bronchial biopsies did not differ between groups (Fig. $2 \mathrm{~b}$ and c). Upon studying different parts of the bronchial biopsies (intact and basal epithelium, smooth muscle, connective tissue) no group differences were found (Additional file 1: Figure S1 and S2).

RAGE levels in healthy never-smokers and smokers are presented in Additional file 1: Table S1.

\section{Associations between COPD, lung function, emphysema and AGEs and RAGE}

Table 3 A-B shows the results of multiple regression analyses with COPD or lung function values as predictors of AGEs and RAGE expression in the different compartments.
In established COPD, a lower $\mathrm{FEV}_{1} \%$ predicted, $\mathrm{FEV}_{1} /$ FVC, FEF25-75 \% predicted, as well as a higher RV/ TLC were associated with a higher SAF, independently of age, gender, number of packyears, BMI, LDL cholesterol and triglycerides (Table 3A). No associations were observed between COPD or lung function values on one hand and AGEs in plasma, sputum and bronchial biopsies on the other hand.

Regarding RAGE, both established COPD and impaired lung function values were associated with higher levels of sRAGE in plasma (Table 3B). In line, a weak but significant negative correlation was shown for the plasma levels of sRAGE with FEV1\% predicted post bronchodilator $\left(r^{2}=0.0943, p=0.0025\right)$. No associations were found of COPD or lung function values with RAGE levels in sputum and bronchial biopsies.

The percentage of the predicted diffusion capacity (DLCO\% predicted) is used as a measure for the amount of emphysema (Table 1). Here, we found a positive correlation between the DLCO\% predicted and the plasma levels of sRAGE $\left(r^{2}=0.186, p<0.0001\right)$ and a negative correlation between the DLCO\% predicted and the SAF levels $\left(r^{2}=\right.$ $0.276, p<0.0001$ ) (Fig. 4). These data indicate that emphysema is associated with low plasma levels of sRAGE and with high levels of accumulated AGEs in the skin.

\section{Associations of AGEs and RAGE expression between different compartments}

Results of multiple regression analyses are presented in Table 4, reflecting associations between AGEs and 

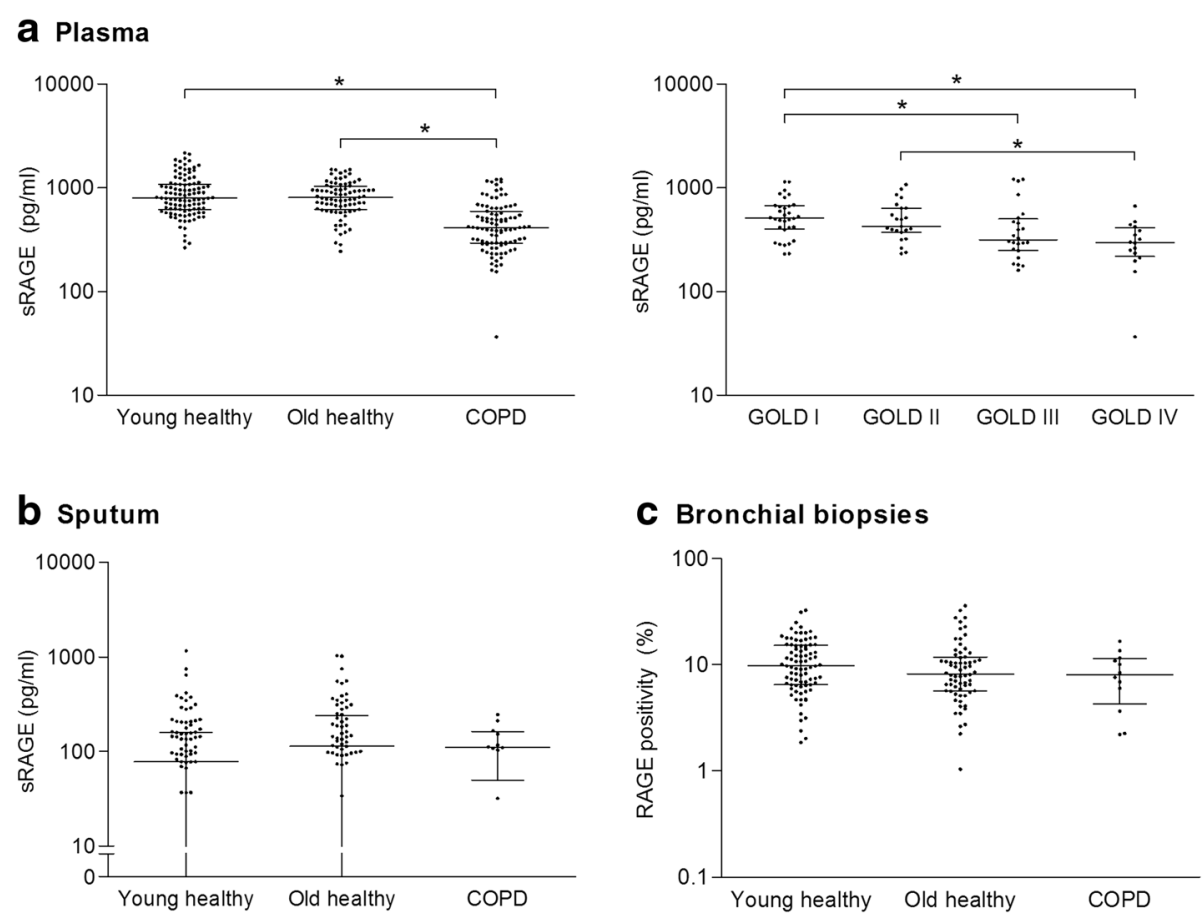

Fig. 2 RAGE expression in plasma, sputum and bronchial biopsies. RAGE levels in a plasma, b sputum, and $\mathbf{c}$ bronchial biopsies. Horizontal lines represent median values with interquartile ranges, ${ }^{*} p<0.05$ between groups

RAGE expression in the different tissues after adjustment for age, gender, packyears, BMI, LDL cholesterol and triglycerides. Lower plasma sRAGE levels were significantly associated with higher SAF values.

\section{Genetic regulation of AGER gene expression levels and RAGE protein levels in sputum}

Cis-eQTL analysis identified a single SNP (rs2071278) within a region of $50 \mathrm{kB}$ flanking the AGER gene, which was associated with gene expression levels of

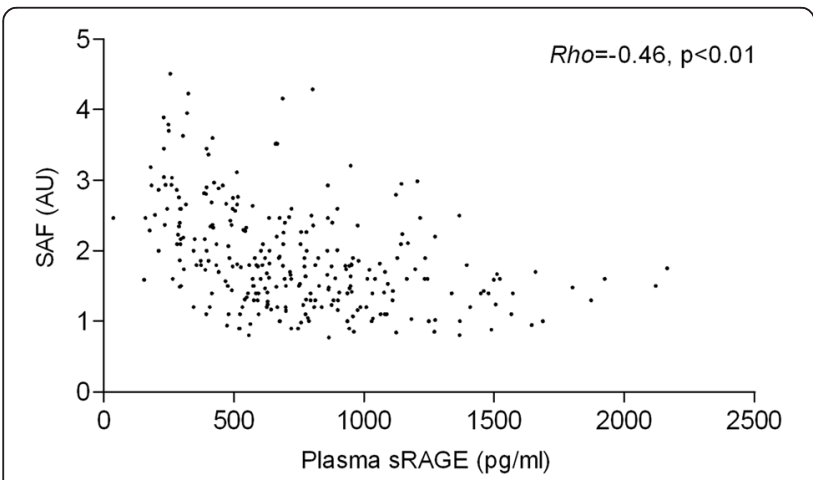

Fig. 3 Associations between SRAGE and SAF. Rho = correlation coefficient, SAF = skin autofluorescence, sRAGE is soluble receptor for advanced glycation endproducts. Association after adjustment for age, gender, packyears, BMI, LDL cholesterol and triglycerides was in $\mathrm{B}=0.00, p=<0.01$
AGER (beta $=-0.109 \pm 0.044, \quad$ FDR $<0.25) \quad$ (Fig. 5a, Additional file 1: Table S3). The SNP was located $13345 \mathrm{bp}$ upstream from the promoter region of AGER. Protein QTL analysis (pQTL) was then run to investigate whether the rs2071278 eQTL influenced the levels of sRAGE detected in sputum and plasma. This analysis showed that rs2071278 regulated the levels of sRAGE measured in sputum (beta $=-80.05 \pm 30.80, p=0.01$ ), with the minor allele leading to decreased levels of sRAGE (Fig. 5b). No interaction was found between rs2071278 and sRAGE levels measured in plasma (beta $=47.62 \pm 59.58, p=0.425$ ). Finally, we investigated whether levels of AGE accumulation in the skin were genetically regulated. A SNP association analysis was conducted on the SAF levels. From this

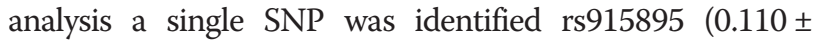
0.038 , FDR < 0.25), 38118 bp upstream of the AGER gene, which is significantly associated with AGE accumulation in the skin (Fig. 5c, Additional file 1: Table S4). Of interest, rs2071278 has a trend for significance (beta $=0.089 \pm 0.049$, $p=0.072$ ), with the minor allele leading to increased SAF levels in the skin (Fig. 5d).

\section{Discussion}

In this study we investigated a large COPD and nonCOPD control population with respect to the accumulation of AGEs and the expression of its receptor RAGE in different body compartments, including plasma, induced sputum, bronchial biopsies and the skin. COPD 
Table 3 Associations of AGEs and RAGEs with COPD and lung function in the total population

\begin{tabular}{|c|c|c|c|c|c|c|c|c|c|c|c|}
\hline \multicolumn{12}{|c|}{ A. Associations of AGEs with COPD and lung function } \\
\hline \multirow[t]{3}{*}{ Dependent variable } & & \multicolumn{10}{|c|}{ Predictor variables } \\
\hline & & \multicolumn{2}{|c|}{ COPD, n/y } & \multicolumn{2}{|c|}{ FEV1, \% predicted } & \multicolumn{2}{|c|}{ FEV1/FVC (\%) } & \multicolumn{2}{|c|}{ RV/TLC (\%) } & \multicolumn{2}{|c|}{ FEF $25-75, \%$ predicted } \\
\hline & & $\beta$ & $p$-value & $\beta$ & $p$-value & $\beta$ & $p$-value & $\beta$ & $p$-value & $\beta$ & $p$-value \\
\hline \multirow[t]{3}{*}{ Plasma } & CEL & 0.009 & 0.924 & -0.465 & 0.642 & 0.581 & 0.562 & 0.117 & 0.204 & 0.0 .41 & 0.635 \\
\hline & CML & 0.093 & 0.327 & -0.081 & 0.283 & -0.088 & 0.337 & 0.052 & 0.572 & -0.027 & 0.761 \\
\hline & Pentosidine & -0.035 & 0.711 & -0.020 & 0.788 & 0.069 & 0.451 & 0.093 & 0.314 & -0.011 & 0.901 \\
\hline \multirow[t]{3}{*}{ Sputum } & CEL & 0.093 & 0.311 & -0.139 & 0.101 & -0.044 & 0.691 & 0.11 & 0.353 & -0.059 & 0.518 \\
\hline & CML & 0.029 & 0.757 & -0.030 & 0.728 & 0.043 & 0.706 & 0.021 & 0.862 & 0.000 & 0.998 \\
\hline & Pentosidine & -0.025 & 0.792 & -0.124 & 0.152 & 0.022 & 0.844 & -0.024 & 0.841 & -0.053 & 0.568 \\
\hline Bronchial biopsies & AGE positivity & -0.065 & 0.550 & 0.139 & 0.155 & 0.075 & 0.551 & -0.126 & 0.420 & 0.094 & 0.379 \\
\hline Skin & SAF & 0.426 & $<0.001$ & -0.302 & $<0.001$ & -0.357 & $<0.001$ & 0.265 & $<0.001$ & -0.347 & $<0.001$ \\
\hline
\end{tabular}

B. Associations of RAGE with COPD and lung function

Dependent variables

Predictor variables

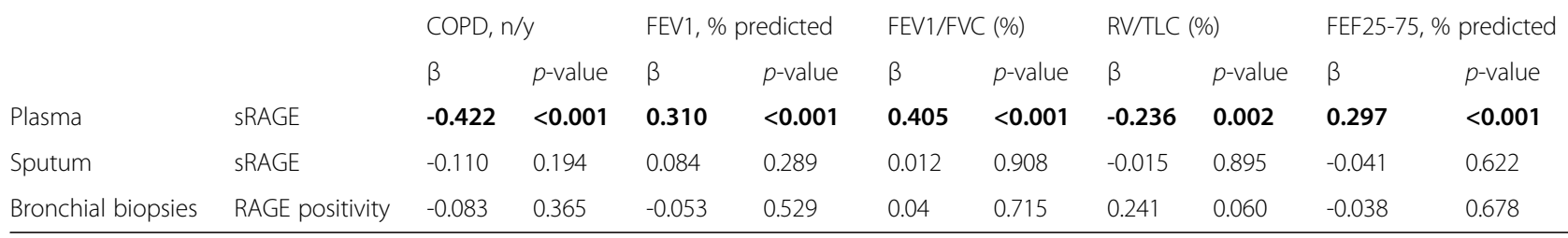

Values in bold represent significant associations. $\beta=$ standardized regression coefficient for predictor variables. Models in Table $A$ were adjusted for age, gender, packyears, BMI, LDL cholesterol and triglycerides; models in Table B were adjusted for age, gender and packyears

$F E V$, forced expiratory volume in one second, FVC forced expiratory volume, $R V$ residual volume, $T L C$ total lung capacity, FEF forced expiratory flow,

$C E L \mathrm{~N}^{\varepsilon}$-(carboxyethyl)lysine, $C M L \mathrm{~N}^{\varepsilon}$-(carboxymethyl)lysine, $A G E s$ advanced glycation endproducts, SAF skin autofluorescence, RAGE receptor for advanced glycation endproducts

has been associated with chronic oxidative stress, the most important accelerator of AGE formation. Our study shows that SAF values in the skin were higher in COPD patients compared to young and old non-COPD controls, whereas the expression of AGEs in bronchial biopsies was not different between the groups. In addition, sRAGE levels in plasma were lower in COPD patients. Of interest, lower sRAGE was associated with higher SAF, fitting the hypothesis of a 'protective' function of sRAGE by acting as a decoy-receptor preventing accumulation of AGEs in the skin.

In COPD, oxidative stress is thought to be continuously increased as a consequence of ongoing inflammation (endogeneous component) and chronic smoking (exogeneous component). This continuous exposure to oxidative stress, both locally in lung tissue as well as systemically in peripheral blood, might lead to increased accumulation of AGEs inside and outside the lung. In the current study we
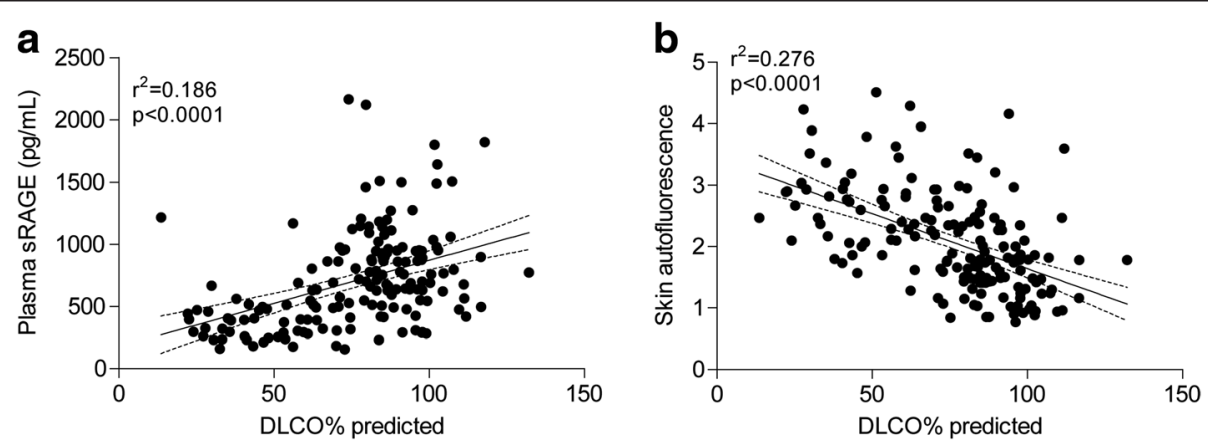

Fig. 4 Associations of DLCO\% predicted with (a) plasma sRAGE levels and (b) skin autofluorescence. All participants from the study were added, including young healthy controls, old healthy controls and COPD patients. Significance was tested using a linear regression analysis. Dotted lines indicate the $95 \%$ confidence intervals 
Table 4 Associations between AGEs and RAGE expression in different tissues

\begin{tabular}{|c|c|c|c|c|c|c|c|c|c|c|c|c|c|c|c|c|c|c|c|c|c|c|c|}
\hline & & \multicolumn{8}{|c|}{ Plasma } & \multicolumn{8}{|c|}{ Sputum } & \multicolumn{4}{|c|}{ Bronchial biopsies } & \multirow{2}{*}{\multicolumn{2}{|c|}{$\begin{array}{l}\text { Skin } \\
\text { SAF }\end{array}$}} \\
\hline & & \multicolumn{2}{|l|}{$\overline{C E L}$} & \multicolumn{2}{|l|}{ CML } & \multicolumn{2}{|c|}{ Pentosidine } & \multicolumn{2}{|l|}{ sRAGE } & \multicolumn{2}{|l|}{ CEL } & \multicolumn{2}{|l|}{ CML } & \multicolumn{2}{|c|}{ Pentosidine } & \multicolumn{2}{|l|}{ sRAGE } & \multicolumn{2}{|c|}{ AGE positivity } & \multicolumn{2}{|c|}{ RAGE positivity } & & \\
\hline \multicolumn{2}{|c|}{ Predictor variables: } & $B$ & $p$ & $B$ & $p$ & $B$ & $p$ & B & $p$ & $B$ & $p$ & B & p & $B$ & $p$ & $B$ & $p$ & $B$ & $p$ & $B$ & $p$ & $B$ & $p$ \\
\hline \multirow[t]{4}{*}{ Plasma } & CEL & - & - & 0.42 & 0.34 & -0.39 & 0.27 & 3.21 & 0.47 & 0.07 & 0.46 & 0.99 & 0.20 & 0.02 & 0.59 & -3.70 & 0.21 & 0.43 & 0.08 & 0.28 & 0.04 & 0.00 & 0.91 \\
\hline & CML & 0.01 & 0.34 & - & - & 0.11 & 0.07 & -1.01 & 0.13 & 0.02 & 0.22 & -0.13 & 0.34 & 0.00 & 0.77 & 0.51 & 0.28 & 0.07 & 0.03 & 0.00 & 0.78 & 0.00 & 0.72 \\
\hline & Pentosidine & -0.02 & 0.27 & 0.13 & 0.07 & - & - & -1.84 & 0.01 & 0.04 & 0.01 & -0.13 & 0.38 & 0.01 & 0.44 & 0.46 & 0.37 & 0.04 & 0.20 & -0.01 & 0.51 & 0.00 & 0.81 \\
\hline & SRAGE & 0.00 & 0.47 & -0.01 & 0.13 & -0.01 & 0.01 & - & - & 0.00 & 0.21 & -0.01 & 0.57 & 0.00 & 0.46 & 0.04 & 0.30 & -0.01 & 0.09 & 0.00 & 0.64 & 0.00 & $0.00^{*}$ \\
\hline \multirow[t]{4}{*}{ Sputum } & CEL & 0.06 & 0.46 & 0.62 & 0.22 & 1.14 & 0.01 & -6.92 & 0.21 & - & - & 2.93 & $0.00^{*}$ & 0.17 & $0.00^{*}$ & -3.30 & 0.23 & 0.51 & 0.01 & -0.07 & 0.53 & 0.00 & 0.91 \\
\hline & CML & 0.01 & 0.20 & -0.05 & 0.34 & -0.04 & 0.38 & -0.33 & 0.57 & 0.03 & $0.00^{*}$ & - & - & 0.00 & 0.50 & -0.63 & 0.03 & 0.03 & 0.07 & 0.00 & 0.72 & 0.00 & 0.51 \\
\hline & Pentosidine & 0.10 & 0.59 & -0.25 & 0.77 & 0.58 & 0.44 & 6.77 & 0.46 & 0.47 & $0.00^{*}$ & 0.92 & 0.50 & - & - & -5.94 & 0.21 & 0.12 & 0.70 & -0.19 & 0.27 & -0.01 & 0.50 \\
\hline & sRAGE & 0.00 & 0.21 & 0.02 & 0.28 & 0.01 & 0.37 & 0.18 & 0.30 & 0.00 & 0.23 & -0.06 & 0.03 & 0.00 & 0.21 & - & - & 0.00 & 0.96 & 0.00 & 0.95 & 0.00 & 0.63 \\
\hline Bronchial & AGE positivity & 0.06 & 0.08 & 0.59 & 0.03 & 0.33 & 0.20 & -4.99 & 0.09 & 0.14 & 0.01 & 0.92 & 0.07 & 0.01 & 0.70 & 0.09 & 0.96 & - & - & 0.10 & 0.07 & 0.00 & 0.39 \\
\hline biopsies & RAGE positivity & 0.13 & 0.04 & -0.13 & 0.78 & -0.29 & 0.51 & -2.52 & 0.64 & -0.06 & 0.53 & 0.32 & 0.72 & -0.06 & 0.27 & -0.19 & 0.95 & 0.30 & 0.07 & - & - & 0.00 & 0.85 \\
\hline Skin & SAF & -0.10 & 0.91 & -1.50 & 0.72 & 0.91 & 0.81 & -154.97 & $0.00^{*}$ & 0.18 & 0.91 & 9.76 & 0.51 & -0.62 & 0.50 & 23.76 & 0.63 & 3.15 & 0.39 & 0.37 & 0.85 & - & - \\
\hline
\end{tabular}

$\mathrm{B}=$ regression coefficient for predictor variables. All models are adjusted for age, gender, packyears, BMI, LDL cholesterol, and triglycerides. * significant $p$-value after Benjamini Hochberg correction for multiple testing CEL N $N^{\varepsilon}$-(carboxyethyl)lysine, CML NE -(carboxymethyl)lysine, SRAGE soluble receptor for advanced glycation endproducts, AGE advanced glycation end products, RAGE receptor for advanced glycation endproducts, SAF skin autofluorescestir 

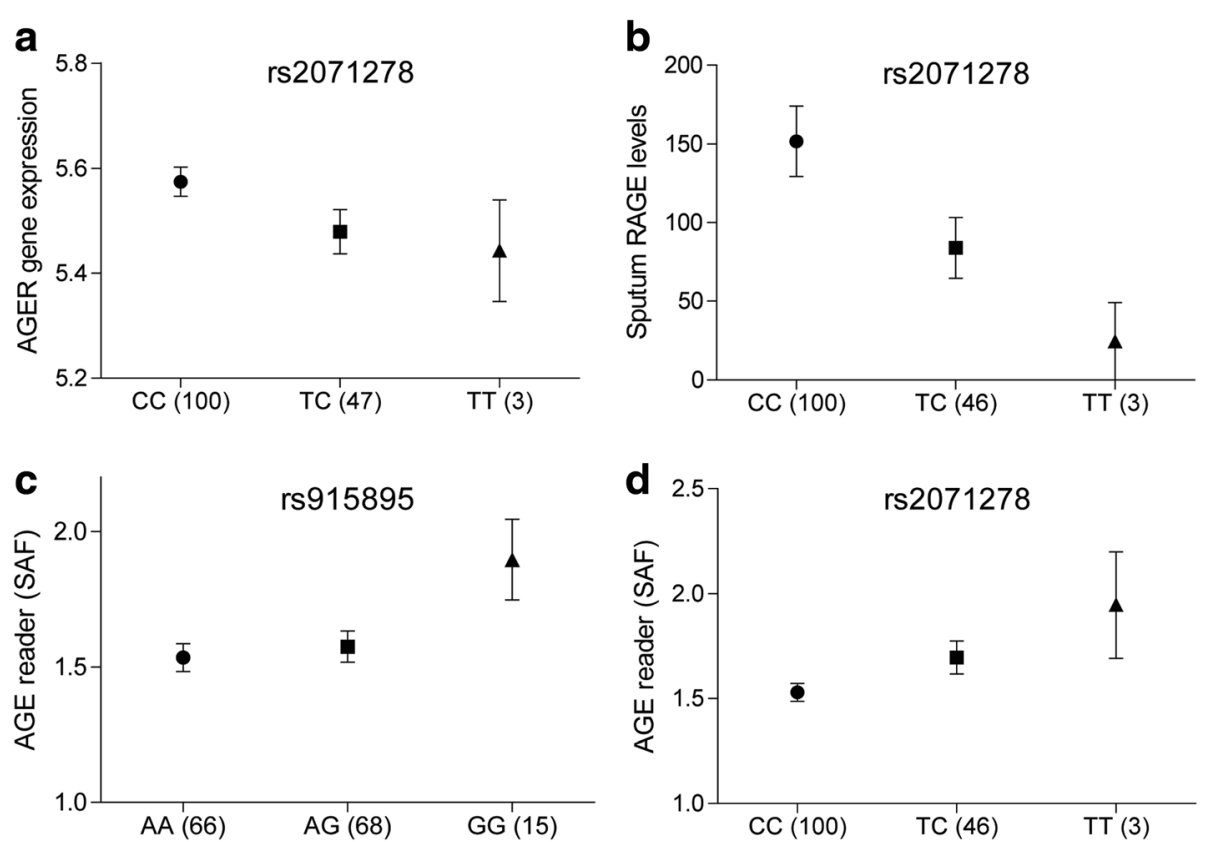

Fig. 5 Genetic regulation of AGER gene expression levels, RAGE protein levels in sputum and AGE levels in skin. $\mathbf{a}$ Expression QTL analysis and $\mathbf{b}$ Protein QTL analysis of the AGER gene expression in bronchial biopsies and levels of soluble RAGE in sputum, respectively. SNP association study with AGE levels detected in the skin with c rs915895 and d rs2071278

demonstrated that the accumulation of AGEs was elevated in the skin of COPD patients, a finding that we and others have observed before [18, 19]. Interestingly, SAF values did not differ significantly between the different severity stages of COPD. This suggests that the formation of AGEs is not increased during disease progression, but may be accelerated in the induction phase of COPD. Our data suggests that AGEs accumulate to some extent during ageing due to oxidative stress responses, whereas this is accelerated with smoking. The highest levels of AGEs would be expected in 'susceptible' smokers who develop COPD over time. Here, AGEs accumulate due to a combination of ageing and disease-related exaggerated responses to smoking and associated local and systemic oxidative stress. Together, our data shows that skin autofluorescence is significantly increased in COPD patients, without significant differences between the different severity stages of COPD.

In contrast with our findings in the skin, the expression of AGEs in bronchial biopsies was not different between COPD patients and non-COPD controls and did not associate with lung function values in the total population. This contradicts a previous study showing higher expression of AGEs in the lung parenchyma and small airways of COPD patients as compared to nonCOPD controls [9]. In an effort to replicate these findings we analyzed immunopositivity of our bronchial biopsies in several ways, e.g. by quantifying AGEs in different parts of the bronchial biopsies and by using different antibodies (against total amount of AGEs, CML and pentosidine). However, no differential expression between the groups was observed. There are several explanations for our negative findings in bronchial biopsies. First, we collected biopsies from the central airways whereas oxidative stress might predominantly exist in the peripheral airways, and thereby also the formation of AGEs. Unfortunately, studies comparing oxidative stress in central and peripheral airways are scarce. One study showed that isoprostane levels in epithelial lining fluid (ELF) from the peripheral airways were higher compared to the central airways, both in smokers with and without airway obstruction [23]. Furthermore, we measured the expression of AGEs in peripheral lung tissue sections of smokers and non-smokers with and without COPD but did not find differences between these (small) groups. Secondly, accumulation of AGEs in the lung might be limited because of the relatively high turn-over rate of cells and extracellular matrix [24]. Finally, a quantification problem may contribute to a lower expression of AGEs in the lungs of COPD patients, as extracellular matrix proteins are reduced in the central airways of COPD patients [25]. Obviously, more research aimed at the accumulation of AGEs is needed in both the central and peripheral airways before definitive conclusions can be drawn regarding our conflicting results.

In plasma, we demonstrated that CML, CEL and pentosidine levels were comparable between COPD patients and non-COPD controls, after correction for confounding 
factors. Our results are in line with three previous studies in COPD investigating plasma CML levels and showing no differences between COPD and non-COPD controls $[14,16,26]$. In contrast, one study showed similar pentosidine levels, but lower CML levels and higher CEL levels in COPD patients [19]. The latter is surprising since CML and CEL are both formed by the same pathway, namely via reactive carbonyl compounds. One explanation might be that the quantification was performed using a different technique, i.e. mass spectrometry. Moreover, AGEs are very volatile, hence a measurement represents a 'snap shot' in time and results can be affected by food intake and smoking as well $[1,27]$.

Besides their harmful local effects in tissue, AGEs can interact with RAGE, thereby triggering intracellular signaling in pro-inflammatory pathways. Two previous studies showed that immunostaining of RAGE was increased in bronchial biopsies and in lung parenchyma of COPD patients $[9,10]$. However, we observed no differences when comparing COPD with non-COPD controls in the current study. RAGE also exists as a soluble form, generated as a splice variant of the advanced glycosylation end product-specific receptor $(A G E R)$ gene or by proteolysis of the receptor from the cell surface. In line with previous studies [13-17], we demonstrated lower levels of sRAGE in plasma of COPD patients and these reduced levels were associated with lower lung function values in the total population. Importantly, we demonstrated for the first time that lower sRAGE levels were associated with increased SAF values, indicating that sRAGE acts as a decoy receptor. The binding of AGEs to sRAGE may induce the clearance of AGEs, thereby preventing the accumulation in body tissues. There are indications that lower sRAGE levels are genetically determined, as a single nucleotide polymorphism (SNP) in the AGER gene associates with lower sRAGE levels [13]. In this perspective, impaired sRAGE levels might contribute to higher levels of AGEs in tissues specifically in COPD.

Furthermore, we assessed the levels of AGEs and RAGE in sputum supernatant, which has not been studied in COPD before. We hypothesized that the sputum levels of AGEs and RAGE might reflect their expression in the lung. No differential levels of both AGEs and RAGE in induced sputum from COPD patients and non-COPD controls were found, nor associations with expression in bronchial biopsies. This observation fits with the similar expression of AGEs and RAGE between COPD and healthy individuals as observed in our bronchial biopsies. An explanation may be that AGEs are released in more peripheral airways and are not captured in sputum, as one study in COPD demonstrated that CML was elevated in ELF collected in the peripheral airways, but not in the central airways [28].
Finally, we have identified a novel SNP, rs2071278, which regulates both gene and protein expression of RAGE. Moreover, we also identified a SNP, rs2071278, showing a reversed association with the accumulation of AGEs in the skin. Further studies are planned to further elucidate the function of this SNP in the regulation of RAGE expression and its role in COPD patients.

This study is unique because of its large population of healthy smokers and never-smokers and a large group of COPD patients of all severities, as well as the availability of different tissues from each participant. In this study, only for 12 out of the 97 COPD patients sputum samples and bronchial biopsies were available, which limited the statistical power of this part of the study. However, even in these 12 patients we have shown a clear trend $(p=0.064)$ for CEL levels in induced sputum to be higher in COPD patients compared to both young and old healthy controls (Table 2). Another limitation is that our study had a cross-sectional design. Longitudinal studies are needed to investigate changes in AGEs and RAGE levels in the different tissues over time and to further assess the potential contributing role of AGEs and RAGE in the development of COPD. The accumulation of AGEs in the skin was assessed using the non-invasive AGE-reader (DiagnOptics Technologies B.V., Groningen, The Netherlands), which has shown to produce reliable values that strongly correlate with the levels of several non-auto-fluorescent AGEs measured in skin biopsies $[21,22]$. However, there are some limitations to this technique, as the AGE-reader is unable to quantify specific AGEs and only estimates the group value of all AGEs present in the skin. Furthermore, also non-AGErelated auto-fluorescent proteins present in the skin can be detected by the AGE-reader.

\section{Conclusions}

To summarize, the role of the AGEs-RAGE pathway in COPD is emerging. Our study contributes to this insight since we show an increased accumulation of AGEs in the skin of COPD patients compared to non-COPD smokers and never-smokers. Moreover, we did not observe differences between COPD and non-COPD controls in central bronchial biopsies, indicating that accumulation of AGEs is not similar in different body compartments. No further associations were found between AGEs and RAGE in the different compartments that were investigated. Interestingly, we demonstrated that lower sRAGE levels associate with higher AGE accumulation in the skin. This fits the hypothesis of a 'protective' function of sRAGE by acting as a decoy-receptor preventing accumulation in the skin. Lastly, we identified novel SNPs associated with both the gene and protein expression of RAGE and the accumulation of AGEs in the skin. 


\section{Additional file}

\section{Additional file 1: Supplementary methods. Sputum induction} bronchial biopsies, skin auto-fluorescence. Supplementary Table 1. AGE and RAGE expression in young and old never-smokers and smokers, and COPD GOLD stages. Supplementary Table 2. Correlations of AGEs and RAGE between different compartments. Supplementary Table 3. Expression QTL analysis of AGER. Supplementary Table 4. SNP association with AGE levels detected in the skin. Supplementary Figure 1.

Quantitative analyses of AGEs (left panel) and RAGE (right panel) expression in A) intact epithelium, B) basal epithelium, C) smooth muscle, D) connective tissue of bronchial biopsies. Intensity of staining was scored by a 4-points scale: $0=$ negative staining, $1=$ weak positive, $2=$ positive, and $3=$ strong positive. Horizontal bars represent median values. Supplementary Figure 2. Representative immunohistological staining of AGEs (AGE 1/750, Cosmo Bio Clone 6D12) and RAGE (RAGE 1/1500, Abcam, ab7764) in young healthy controls, old healthy controls and COPD patients. Pictures are shown as 40x magnification, scans taken using the Hamamatsu Slide Scanner (Hamamatsu Photonics, Hamamatsu City, Japan). Supplementary Figure 3. Quantitative analyses of AGEs (left panel) and RAGE (right panel) expression in A) epithelium and B) smooth muscle of the peripheral airways. Intensity of staining was scored by a 4-points scale: $0=$ negative staining, $1=$ weak positive, $2=$ positive, and $3=$ strong positive. Horizontal bars represent median values.

\section{Competing interests}

The authors declare that they have no competing interests.

\section{Authors' contributions}

Design and conduct of the study: SJMH, AJMVO, WT, DSP, NHTTH. Data collection and analysis: SJMH, SDP, AF, ATLTL, EDT, MVDB, ML, LK, JWJL, $\mathrm{NHTTH}, \mathrm{HMB}$. Data interpretation: SJMH, ATLTL, AF, EDT, MVDB, SDP, LK, JWJL, HMB, AJMVO, ML, WT, DSP, NHTTH. Manuscript writing: SJMH, SDP, AF, $\mathrm{NHTTH}$. All authors have read and approved the final version of the article.

\section{Acknowledgements}

None.

\section{Funding}

NCT00807469 and NCT00850863: T1-108 grant from Top Institute Pharma, with partners Nycomed, GlaxoSmithKline, University Medical Center Groningen (UMCG), and University of Groningen (RUG); NCT00848406: The Royal Netherlands Academy of Arts and Sciences.

\section{Author details}

${ }^{1}$ Department of Pulmonary Diseases, University of Groningen, University Medical Center Groningen, Hanzeplein 1, 9713, GZ, Groningen, The Netherlands. 'University of Groningen, University Medical Center Groningen, GRIAC research institute, Groningen, The Netherlands. ${ }^{3}$ Department of Respiratory Medicine, University Medical Center Utrecht, Utrecht, The Netherlands. ${ }^{4}$ Department of Epidemiology, University of Groningen, University Medical Center Groningen, Groningen, The Netherlands. ${ }^{5}$ Department of Pathology and Medical Biology, University of Groningen, University Medical Center Groningen, Groningen, The Netherlands.

Received: 31 December 2015 Accepted: 19 April 2016 Published online: 26 April 2016

\section{References}

1. Cerami C, Founds H, Nicholl I, Mitsuhashi T, Giordano D, Vanpatten S, Lee A, Al-Abed Y, Vlassara H, Bucala R, Cerami A. Tobacco smoke is a source of toxic reactive glycation products. Proc Natl Acad Sci U S A. 1997;94:13915-20

2. Nicholl ID, Stitt AW, Moore JE, Ritchie AJ, Archer DB, Bucala R. Increased levels of advanced glycation endproducts in the lenses and blood vessels of cigarette smokers. Mol Med. 1998;4:594-601.

3. Singh VP, Bali A, Singh N, Jaggi AS. Advanced glycation end products and diabetic complications. Korean J Physiol Pharmacol. 2014;18:1-14.

4. Monnier VM, Mustata GT, Biemel KL, Reihl O, Lederer MO, Zhenyu D, Sell DR. Cross-linking of the extracellular matrix by the maillard reaction in aging and diabetes: an update on "a puzzle nearing resolution". Ann N Y Acad Sci. 2005;1043:533-44.

5. Bierhaus A, Humpert PM, Morcos M, Wendt T, Chavakis T, Arnold B, Stern DM, Nawroth PP. Understanding RAGE, the receptor for advanced glycation end products.

J Mol Med (Berl). 2005:83:876-86

6. Buckley ST, Ehrhardt C. The receptor for advanced glycation end products (RAGE) and the lung. J Biomed Biotechnol. 2010;2010:917108.

7. Castaldi PJ, Cho MH, Litonjua AA, Bakke P, Gulsvik A, Lomas DA, Anderson W, Beaty TH, Hokanson JE, Crapo JD, Laird N, Silverman EK COPDGene and Eclipse Investigators. The association of genome-wide significant spirometric loci with chronic obstructive pulmonary disease susceptibility. Am J Respir Cell Mol Biol. 2011:45:1147-53.

8. Repapi E, Sayers I, Wain LV, Burton PR, Johnson T, Obeidat M, Zhao JH, Ramasamy A, Zhai G, Vitart V, Huffman JE, Igl W, Albrecht E, Deloukas P, Henderson J, Granell R, McArdle WL, Rudnicka AR, Wellcome Trust Case Control Consortium, Barroso I, Loos RJF, Wareham NJ, Mustelin L, Rantanen T, Surakka I, Imboden M, Wichmann HE, Grkovic I, Jankovic S, Zgaga L, et al. Genome-wide association study identifies five loci associated with lung function. Nat Genet. 2010;42:36-44.

9. Wu L, Ma L, Nicholson LFB, Black PN. Advanced glycation end products and its receptor (RAGE) are increased in patients with COPD. Respir Med. 2011; 105:329-36.

10. Ferhani N, Letuve S, Kozhich A, Thibaudeau O, Grandsaigne M, Maret M, Dombret M-C, Sims GP, Kolbeck R, Coyle AJ, Aubier M, Pretolani M. Expression of high-mobility group box 1 and of receptor for advanced glycation end products in chronic obstructive pulmonary disease. Am J Respir Crit Care Med. 2010;181:917-27.

11. Pouwels SD, Nawijn MC, Bathoorn E, Riezebos-Brilman A, van Oosterhout AJM, Kerstjens HAM, Heijink IH. Increased serum levels of LL37, HMGB1 and S100A9 during exacerbation in COPD patients. Eur Respir J. 2015;45: 1482-5.

12. Pouwels SD, Heijink IH, ten Hacken NHT, Vandenabeele P, Krysko DV, Nawijn $M C$, van Oosterhout AJM. DAMPs activating innate and adaptive immune responses in COPD. Mucosal Immunol. 2014;7:215-26.

13. Cheng DT, Kim DK, Cockayne DA, Belousov A, Bitter $\mathrm{H}$, Cho MH, Duvoix A, Edwards LD, Lomas DA, Miller BE, Reynaert N, Tal-Singer R, Wouters EFM, Agustí A, Fabbri LM, Rames A, Visvanathan S, Rennard SI, Jones P, Parmar H, MacNee W, Wolff G, Silverman EK, Mayer RJ, Pillai SG, TESRA and ECLIPSE Investigators. Systemic soluble receptor for advanced glycation endproducts is a biomarker of emphysema and associated with AGER genetic variants in patients with chronic obstructive pulmonary disease. Am J Respir Crit Care Med. 2013;188: 948-57.

14. Gopal P, Rutten EPA, Dentener MA, Wouters EFM, Reynaert NL. Decreased plasma sRAGE levels in COPD: influence of oxygen therapy. Eur J Clin Invest. 2012:42:807-14.

15. Iwamoto H, Gao J, Koskela J, Kinnula V, Kobayashi H, Laitinen T, Mazur W. Differences in plasma and sputum biomarkers between COPD and COPD-asthma overlap. Eur Respir J. 2014;43:421-9.

16. Miniati M, Monti S, Basta G, Cocci F, Fornai E, Bottai M. Soluble receptor for advanced glycation end products in COPD: relationship with emphysema and chronic cor pulmonale: a case-control study. Respir Res. 2011;12:37.

17. Smith DJ, Yerkovich ST, Towers MA, Carroll ML, Thomas R, Upham JW. Reduced soluble receptor for advanced glycation end-products in COPD. Eur Respir J. 2011;37:516-22.

18. Hoonhorst SJM, Lo Tam Loi AT, Hartman JE, Telenga ED, van den Berge M, Koenderman L, Lammers JWJ, Boezen HM, Postma DS, Ten Hacken NHT. Advanced glycation end products in the skin are enhanced in COPD. Metabolism. 2014;63:1149-56.

19. Gopal P, Reynaert NL, Scheijen JLJM, Engelen L, Schalkwijk CG, Franssen FME, Wouters EFM, Rutten EPA. Plasma advanced glycation endproducts and skin autofluorescence are increased in COPD. Eur Respir J. 2014;43:430-8.

20. Lo Tam Loi AT, Hoonhorst SJM, Franciosi L, Bischoff R, Hoffmann RF, Heijink ! van Oosterhout AJM, Boezen HM, Timens W, Postma DS, Lammers J-W, Koenderman L, Ten Hacken NHT. Acute and chronic inflammatory responses induced by smoking in individuals susceptible and non-susceptible to development of COPD: from specific disease phenotyping towards novel therapy. Protocol of a cross-sectional study. BMJ Open. 2013;3. 
21. Meerwaldt R, Links T, Graaff R, Thorpe SR, Baynes JW, Hartog J, Gans R, Smit A. Simple noninvasive measurement of skin autofluorescence. Ann N Y Acad Sci. 2005;1043:290-8.

22. Meerwaldt R, Graaff R, Oomen PHN, Links TP, Jager JJ, Alderson NL, Thorpe SR, Baynes JW, Gans ROB, Smit AJ. Simple non-invasive assessment of advanced glycation endproduct accumulation. Diabetologia. 2004;47:1324-30.

23. Kodama T, Kanazawa H, Tochino Y, Kyoh S, Asai K, Hirata K. A technological advance comparing epithelial lining fluid from different regions of the lung in smokers. Respir Med. 2009;103:35-40.

24. Rawlins EL, Hogan BLM. Epithelial stem cells of the lung: privileged few or opportunities for many? Development. 2006;133:2455-65.

25. Annoni R, Lanças T, Yukimatsu Tanigawa R, de Medeiros Matsushita M de Morais Fernezlian S, Bruno A, Fernando Ferraz da Silva L, Roughley PJ, Battaglia S, Dolhnikoff M, Hiemstra PS, Sterk PJ, Rabe KF, Mauad T. Extracellular matrix composition in COPD. Eur Respir J. 2012;40:1362-73.

26. Boschetto P, Campo I, Stendardo M, Casimirri E, Tinelli C, Gorrini M, Ceconi C, Fucili A, Potena A, Papi A, Ballerin L, Fabbri LM, Luisetti M. Plasma sRAGE and $\mathrm{N}$-(carboxymethyl) lysine in patients with CHF and/or COPD. Eur J Clin Invest. 2013:43:562-9

27. Uribarri J, Cai W, Sandu O, Peppa M, Goldberg T, Vlassara H. Diet-derived advanced glycation end products are major contributors to the body's AGE pool and induce inflammation in healthy subjects. Ann N Y Acad Sci. 2005;1043:461-6.

28. Kanazawa H, Kodama T, Asai K, Matsumura S, Hirata K. Increased levels of $\mathrm{N}$ (epsilon)-(carboxymethyl)|ysine in epithelial lining fluid from peripheral airways in patients with chronic obstructive pulmonary disease: a pilot study. Clin Sci (Lond). 2010;119:143-9.

\section{Submit your next manuscript to BioMed Central and we will help you at every step:}

- We accept pre-submission inquiries

- Our selector tool helps you to find the most relevant journal

- We provide round the clock customer support

- Convenient online submission

- Thorough peer review

- Inclusion in PubMed and all major indexing services

- Maximum visibility for your research

Submit your manuscript at www.biomedcentral.com/submit

C Biomed Central 